\title{
La innovación social en el contexto de la responsabilidad social empresarial
}

\author{
María de Fátima León / leonfati@ula.ve \\ Universidad de los Andes \\ Mérida, Venezuela \\ María Virginia Baptista / maria.baptista@ula.ve \\ Universidad de los Andes \\ Mérida, Venezuela \\ Hernán Contreras / hcontreras@ceo-consultores.com \\ Universidad Católica Andrés Bello \\ Caracas, Venezuela
}

Recibido: 8 de agosto de 2011

Aceptado: 30 de abril de 2012

\section{RESUMEN:}

Ante una realidad caracterizada por los problemas sociales y ambientales no resueltos, es común observar el comportamiento de las empresas en función de su contribución en la solución o tratamiento de estos problemas. Muchas de estas iniciativas son ejemplos de innovaciones sociales que proponen productos nuevos, procesos y relaciones para beneficiar a los grupos más desfavorecidos en temas como seguridad, salud, educación y ambiente, entre otros. En este sentido, esta investigación documental aborda el papel de la innovación social en el marco de la responsabilidad social empresarial para lo cual se realiza un recorrido teórico por el tema de la innovación, innovación social y Responsabilidad Social Empresarial. Asimismo, a través del tamiz de lo que se puede considerar innovación social se plantean algunos ejemplos de empresas venezolanas que con un enfoque socialmente responsable se encaminan hacia la madurez hacia una empresa socialmente ética.

Palabras clave: innovación, innovación social, responsabilidad social empresarial

\section{ABSTRACT:}

Faced with a reality characterized by unsolved social and environmental problems, it is common to observe the behavior of firms in terms of its contribution in the resolution or treatment of these problems. Many of these initiatives are examples 
of social innovations offering new products, processes and relationships in terms of benefiting the most disadvantaged groups in areas such as safety, health, education, environment, among others. In this sense, this documentary research examines the role of social innovation in the context of corporate social responsibility, through a review of theoretical topic of innovation, social innovation and corporate social responsibility. Also, through the filter of what can be considered social innovation, raises some examples of Venezuelan companies with socially responsible approaches moving toward maturity in a socially ethical enterprise.

Keywords: innovation, social innovation, corporate social responsibility, value proposition

\section{INTRODUCCIÓN}

En entornos cada vez más complejos donde prevalecen problemas económicos, sociales y ambientales que obstaculizan el desarrollo de las sociedades, el sector empresarial se enfrenta a grandes retos en función de la contribución que debe ofrecer a las localidades donde se asientan. En este sentido, cobra importancia la innovación empresarial, no sólo la relativa a la generación de productos, servicios y procesos que logren mayores cuotas de mercado y rentabilidad. En esta realidad, el enfoque social es esencial y la innovación empresarial debe transcender lo meramente económico y apostar por su contribución con el desarrollo de las sociedades a través de innovaciones sociales.

En otras palabras, la innovación incide en las diversas funciones y procesos organizacionales que marcan ventajas en cuanto a productividad y competitividad, en aspectos que trascienden los objetivos corporativos y que la ubican como una fuerza o variable determinante en el desarrollo de las comunidades donde se desenvuelve y progresa. Este pensamiento es uno de los elementos claves que impulsa el movimiento de la responsabilidad social empresarial (RSE).

En este orden de ideas, este artículo, presenta una investigación documental que se desarrolló con el propósito de resaltar la función de la actividad innovadora en responsabilidad social por parte de las organizaciones. Es importante conocer los avances que se han desarrollado a nivel mundial con relación al tema de la innovación y de esta en función de la responsabilidad social de las empresas. Uno 
de los intereses principales de la investigación ha sido conocer las experiencias de innovación socialmente responsables que existen en Venezuela. En este contexto, se parte de las coincidencias entre los autores en función de lo que debe considerarse como innovación social y a través de este tamiz se muestran algunos ejemplos de acciones empresariales que apuestan en este sentido.

\section{INNOVACIÓN: DEFINICIÓN Y CONTEXTUALIZACIÓN}

Innovación es la introducción de un nuevo o significativamente mejorado producto, servicio, proceso o método de comercialización $\mathrm{u}$ organizativo reflejado en prácticas internas de la empresa, organización del lugar de trabajo o relaciones exteriores (OCDE, 2006). Asimismo, el desarrollo de los países está relacionado con la innovación, la cual requiere la participación de diferentes elementos, aspectos que se vinculen en un mismo sistema, tecnología, empresas o instituciones. Se habla entonces de sistemas de innovación que pueden darse en espacios geográficos nacionales, sectoriales, regionales o locales. En este sentido, importantes instituciones internacionales, gobiernos y empresas se preocupan por el fomento de la innovación. La Comisión Europea, Organización para la Cooperación y el Desarrollo Económico (OECD), Programa de Naciones Unidas para el Desarrollo (PNUD) y el Banco Interamericano de Desarrollo (BID), entre otros, han desarrollado estrategias que contribuyen al logro de ambientes innovadores.

Una iniciativa que busca el fomento de la innovación es el Manual de Oslo, que surge del acuerdo de los países que componen la Organización para la Cooperación y el Desarrollo Económico (OCDE, 2006) y proporciona las directrices para la recopilación, interpretación y aplicación de datos sobre innovación y su relación con el desarrollo económico. Este documento recalca la vinculación entre las empresas e instituciones en el proceso de innovación y la importancia de ésta en los sectores de baja intensidad en investigación y desarrollo, así como en empresas e industrias de escaso contenido tecnológico. Además, menciona algunos tipos de innovación, los cuales pueden ser: de productos, procesos, mercadotecnia u organización. 
De igual forma, el Manual reconoce la importancia de los factores no tecnológicos como potenciadores de la capacidad innovadora de las empresas y prioriza en la investigación y el desarrollo como determinantes en los procesos de innovación. Sin embargo, gran parte de ésta proviene del personal calificado, de las relaciones con otras organizaciones y de una estructura organizacional que fomente el aprendizaje y el conocimiento (OCDE, 2006).

Por supuesto, analizar la innovación desde el contexto de la práctica de países europeos tiene sus dificultades de aplicación en nuestros países en vías de desarrollo, por lo cual, se realizaron adaptaciones que hicieran más práctica y útil la recopilación e interpretación de la innovación en los países menos avanzados. Surge así el Manual de Bogotá. Este manual nace de la iniciativa de la Red Iberoamericana de Indicadores de Ciencia y Tecnología (RICYT, 2001). En agosto de 2000 se presentó el proyecto de "Normalización de indicadores de innovación tecnológica en América Latina”, el cual contó con el apoyo financiero de la Organización de los Estados Americanos (OEA). Su cometido se fundamenta en favorecer la realización de estudios sobre los procesos de innovación tecnológica en la región y a incrementar las capacidades de los países de América Latina para la construcción de indicadores de innovación que resulten comparables entre sí y con los producidos globalmente. En marzo de 2001 se publica el Manual de Bogotá que recoge las conclusiones más importantes de la discusión realizada sobre innovación en la región.

En el Manual de Bogotá se reseñan algunos factores que pueden afectar la innovación en los países en desarrollo, entre los cuales se destacan los económicos (riesgos, costos, falta de fuentes de financiamiento), empresariales (información sobre tecnología, falta de potencial innovador, resistencia al cambio, deficiencia en la consecución de servicios externos) y otros factores, como la falta de oportunidad tecnológica y de infraestructura, la debilidad en los derechos de propiedad, el marco legal y tributación, la poca o nula necesidad de innovar y la escasa respuesta de los clientes a los nuevos procesos y productos.

Por otro lado, debe tomarse en cuenta la rápida obsolescencia de los procesos y productos, así como la creciente complejidad 
tecnológica, con lo cual cobra aún más sentido la cooperación entre organizaciones a través de redes o sistemas de innovación. La empresa, para aprovechar el potencial innovador, debe, por un lado, revisar las debilidades y fortalezas propias (formación de sus empleados, sistemas de control de calidad, nuevas tecnologías de información, herramientas estadísticas, redes o sistemas de innovación) y, por otro, conocer las necesidades, tanto de sus consumidores como de las comunidades donde se desarrollan.

\section{De INNOVACIÓN TRADICIONAL A INNOVACIÓN SOCIAL}

La innovación tecnológica, en su sentido más amplio, incluye cambios e innovaciones sociales, e incluso, según Alburquerque (2003), en muchas ocasiones, la competitividad no se logra por la adquisición de nuevas máquinas o tecnología, sino por los cambios sociales y culturales que se producen en la organización. Los cambios sociales dan lugar a redes de comunicación que aúnan esfuerzos y sinergias para aumentar la calidad de prestaciones producto de los incrementos en la productividad; dinamizar el potencial creativo e innovador en la solución de problemas en ambientes propicios y estimulantes; y satisfacer continuamente los cambios en las necesidades de la demanda. En palabras de Llano (2004):

La idea de empresa debe entenderse como esencialmente ligada a la emergencia de lo nuevo. No es preciso insistir en que esta orientación hacia la novedad no consiste en la mera aplicación de las "nuevas tecnologías", porque en la medida en que están disponibles ya no son nuevas. La innovación más característica de las corporaciones empresariales no se refiere tanto a la técnica como al comportamiento humano. La técnica está regida por reglas. En cambio, lo nuevo en la conducta del hombre nunca se agota en el uso de unas reglas ya dadas, sino que se extiende al descubrimiento de normas nuevas y, sobre todo, a ese amplio territorio del trabajo humano en el que no rigen los esquemas abstractos y estereotipados, sino que el acierto viene dado por el ejercicio creativo de la inteligencia y la capacidad de decisión, lo cual requiere estudio, reflexión, diálogo, imaginación, espontaneidad, iniciativa, prudencia, agilidad de decisión, juventud interior. 
En sintonía con lo expuesto, además de la innovación tradicional ${ }^{1}$ se puede también hablar de innovación social, la cual para Phills, Deiglmeier y Miller (2008), es una solución a un problema social que es más eficaz, eficiente y sostenible que las soluciones existentes cuyo valor creado se acumula en la sociedad en su conjunto y no en los particulares. Una innovación social puede ser un producto, proceso de producción o la tecnología, como la innovación en general. También puede ser un principio, una idea, una pieza de legislación, un movimiento social, una intervención o alguna combinación de ellos. Una innovación es verdaderamente social sólo si la balanza se inclina hacia el valor social, es decir, beneficios para la sociedad (o reducción de costos para la sociedad), en lugar de ganancias para las empresas (generar algo más que valor financiero).

Según estos autores, dada la complejidad de los problemas sociales, el carácter social lo detentan tanto el gobierno, como las organizaciones no gubernamentales, lucrativas y no lucrativas. Las organizaciones sin fines de lucro y el gobierno necesitan aprender de la gestión empresarial; el gobierno y las empresas necesitan de la experiencia en problemas sociales que poseen las organizaciones sin fines de lucro; y las empresas y las organizaciones sin fines de lucro necesitan acercarse a los gobiernos para contribuir en la creación de políticas públicas. Estas alianzas son básicas para generar innovaciones sociales e inversiones socialmente responsables, que incluso puedan llegar a ser rentables. Asimismo, aunque pueda existir un debate sobre el carácter social de las innovaciones, hay consenso sobre lo que constituye una necesidad social y la importancia de los objetivos sociales, es decir, la preservación del medio ambiente, la mejoría de salud, la educación y la justicia, entre otros.

\footnotetext{
${ }^{1}$ Si se considera el contexto del Manual de Bogotá, podría analizarse la innovación tradicional a través del tamiz de indicadores relacionados con la investigación y desarrollo (proyectos, empleo inversión y resultados), esfuerzos de innovación (tecnología incorporada y no incorporada al capital), capacitación, modernización organizacional, diseño (productos, procesos, ingeniería de procesos), comercialización (distribución, mercadeo) y resultados de innovación (innovaciones de producto por grado de novedad, de proceso por grado de complejidad, organizacionales, comercialización, relación de la innovación e inversión, impacto económico, entre otros (RYCTY, 2001).
} 
Para los expertos del Future Trends Forum (citado por la Fundación de la Innovación Bankinter, 2009), existen factores de éxito que contribuyen a la innovación social, los cuales están determinados por: a) la innovación planificada y aplicada a los modelos de negocio y productos; b) la habilidad de las empresas privadas para resolver de manera más eficaz que los gobiernos, los problemas sociales y medioambientales; c) la innovación colaborativa como ecosistema del futuro para poner en común las ideas de una mayor variedad de agentes en un entorno abierto a la participación; y d) la responsabilidad de la tecnología (comunicaciones, internet) para facilitar la innovación colaborativa.

De igual forma, consideran el desempeño de la innovación en diferentes aspectos: la disminución de la pobreza (emprendedores sociales y negocios inclusivos), la preservación del medioambiente (tecnologías limpias, escalabilidad de la innovación ambiental y crear conciencia ambiental), la cohesión social (inserción laboral de grupos excluidos, la mujer en el mercado de trabajo, cohesión internauta) y la cooperación y los modelos de negocios innovadores que apuesten a la salud y la educación. Plantean que el
futuro de la innovación social está en empresas capaces de construir organizaciones sostenibles y escalables que puedan entregar bienes y servicios asequibles y críticos para aumentar el nivel de vida de los más desfavorecidos. Se espera que estas organizaciones sostenibles con una clara vocación social encuentren el camino hacia la resolución de los retos del siglo XXI. (Fundación de la Innovación Bankinter, 2009:9)

Para la Comisión Europea (2011ª), la innovación social, en su forma más básica, se relaciona con nuevos procesos (formas de organización y relaciones) y resultados que respondan a las demandas sociales de los grupos más vulnerables no atendidos por las instituciones públicas o el mercado. Un siguiente nivel de evolución de la innovación social, disiparía la frontera entre lo económico y lo social pues lo social se convierte en una oportunidad para generar valor. La innovación es vista como un proceso que debe enfrentar a los retos de la sociedad a través de nuevas formas de relaciones entre los actores sociales. Los principales problemas mundiales, como por ejemplo, el cambio 
climático, el envejecimiento, la migración, el género y la salud, entre otros, son de naturaleza tanto económica como social (no sólo temas de política social, sino también parte integral del desarrollo económico). Otro argumento es que la innovación en el sector social genera productividad y valor económico para la sociedad en su conjunto (bienestar más allá del PIB).

Idealmente, la innovación social en su nivel máximo de desarrollo, llevaría la reforma de la sociedad a un espacio más participativo de empoderamiento, aprendizaje y bienestar. A este nivel, la dimensión social de la innovación se refiere a los cambios en las actitudes, los valores fundamentales, las estrategias, las políticas, las estructuras organizativas y los procesos, los sistemas de entrega y servicios, los métodos y las formas de trabajo, las responsabilidades y las tareas de las instituciones y los vínculos entre ellos y los diferentes participantes, aspectos y variables. Además, la relevancia de la innovación social para la Comunidad Europea $\left(2011^{\mathrm{b}}\right)$, se puede observar en la iniciativa "Unión por la Innovación" la cual tiene como objetivo reforzar la capacidad de las empresas para enfrentar los retos sociales mediante la innovación.

Para Harris y Albury (2009), la innovación social es la inspirada por el bien social y público y el deseo de conocer las necesidades sociales mal servidas u obviadas por el mercado o el estado. La innovación social puede tener lugar dentro o fuera de los servicios públicos, puede ser desarrollada por el sector público, privado o de terceros y necesariamente debe abordar los principales desafíos sociales.

La innovación social puede también ser vista como una iniciativa empresarial centrada en objetivos sociales, productos de calidad, nuevos métodos de organización y/o de producción, gobernabilidad, nuevas relaciones de mercado y nuevas formas jurídicas (Westall, 2007). De acuerdo con NESTA (2008), la innovación plantea nuevos productos, servicios o modelos para cumplir con necesidades básicas insatisfechas. Puede ser transmitida a través de una profesión o sector (educación o salud) o geográficamente de un lugar a otro.

En esta misma línea de pensamiento, para la OCDE (2010), la innovación social busca respuestas nuevas a los problemas sociales a través de la identificación y entrega de productos o servicios nuevos que mejoren la calidad de vida de los individuos y las comunidades; la 
identificación e implementación de nuevos procesos de integración laboral de mercado, nuevas competencias, nuevos puestos de trabajo y nuevas formas de participación. Se trata del bienestar de los individuos, las comunidades, los consumidores y los productores. Se trata de satisfacer las necesidades no provistas por el mercado. La innovación social apuesta por el bienestar de los individuos y la comunidad a través del empleo, el consumo o la participación; en resumen, proporcionar soluciones a los problemas individuales y comunitarios.

En esencia, la innovación no puede considerarse exclusivamente un mecanismo económico o un proceso técnico, pues antes es un fenómeno social a través del cual se expresan las necesidades, la creatividad, la historia, la cultura, la educación, la organización política institucional y económica de una sociedad. Más aún, si consideramos la realidad de los países en vías de desarrollo, es un hecho que existen graves problemas sociales reflejados en los flagelos de la pobreza y desigualdad. No ha sido posible para la mayoría de los gobiernos erradicar estos problemas y las empresas han asumido de alguna forma su resolución ${ }^{2}$. En otras palabras, las deficiencias de su entorno en relación a aspectos como pobreza, infraestructura, salud, educación, vialidad y seguridad, entre otros, puede a la larga ver afectada su sostenibilidad y rentabilidad en el tiempo.

A tenor con lo anterior, es entonces estratégico para su gestión, contribuir con el desarrollo de sus entornos, pues no hacerlo tiene implicaciones negativas para su sostenimiento en el mercado. En concordancia con esta idea, la Comisión Europea (1995) plantea en su Libro Verde de la innovación, que ante los problemas de una sociedad, la innovación puede ser una opción para mejorar la calidad de vida de las comunidades a cualquier nivel: salud, seguridad, transporte, comunicaciones, seguridad en del trabajo y medio ambiente, entre muchos otros beneficios que puede proveer.

\footnotetext{
${ }^{2}$ En Latinoamérica y el Caribe, la inversión social de la empresa, en muchas ocasiones, sustituye el papel que por naturaleza corresponde a los gobiernos. El predominio de la pobreza en América Latina ha llevado a las empresas a través de la inversión social privada, a reemplazar lo que los estados no pueden hacer. Es precisamente la pobreza, uno de los factores impulsores de RSE en la región (Langlois, 2010).
} 
De las visiones antes expuestas, se extraen algunos criterios que pueden considerarse como características de una innovación con carácter social y que se muestran en la Tabla 1.

Tabla 1: Características de la innovación con carácter social

\begin{tabular}{|c|c|}
\hline Propuesta & Características \\
\hline $\begin{array}{l}\text { Alburquerque } \\
\text { (2003) }\end{array}$ & $\begin{array}{l}\text { Aumentar la calidad de prestaciones, producto de los } \\
\text { incrementos en la productividad; dinamizar el potencial creativo } \\
\text { e innovador en la solución de problemas en ambientes propicios } \\
\text { y estimulantes; satisfacer los cambios en las necesidades de la } \\
\text { demanda. }\end{array}$ \\
\hline $\begin{array}{l}\text { Comunidad } \\
\text { Europea }\left(2011^{a}\right)\end{array}$ & $\begin{array}{l}\text { Proceso y resultados que respondan a las demandas sociales } \\
\text { de los grupos más vulnerables no atendidos por las instituciones } \\
\text { públicas o el mercado. Imbricación de lo económico y social. } \\
\text { Proceso participativo de empoderamiento, aprendizaje } \\
\text { y bienestar; implica cambios en las actitudes, valores } \\
\text { fundamentales, estrategias, políticas, estructuras organizativas } \\
\text { y procesos, sistemas de entrega y servicios, métodos y formas } \\
\text { de trabajo, responsabilidades y tareas de las instituciones y los } \\
\text { vínculos entre los diferentes actores }\end{array}$ \\
\hline $\begin{array}{l}\text { Comisión } \\
\text { Europea (1995) }\end{array}$ & $\begin{array}{l}\text { Calidad de vida de las comunidades a cualquier nivel, salud, } \\
\text { seguridad, transporte, comunicaciones, seguridad en del trabajo } \\
\text { y medio ambiente, entre muchos otros beneficios }\end{array}$ \\
\hline $\begin{array}{l}\text { Phills, Deiglmeier } \\
\text { y Miller (2008) }\end{array}$ & $\begin{array}{l}\text { Mejora de productos, procesos de producción, tecnologías, } \\
\text { ideas, legislaciones, movimiento social, una intervención en la } \\
\text { comunidad, o alguna combinación. Solución a un problema social } \\
\text { que es más eficaz, eficiente y sostenible que las soluciones } \\
\text { existentes y el valor creado se acumula principalmente en } \\
\text { la sociedad (beneficios o reducción de costos). Intervienen } \\
\text { gobiernos y organizaciones con o sin fines del lucro. }\end{array}$ \\
\hline $\begin{array}{l}\text { Future } \\
\text { Trends Forum } \\
\text { (Fundación de } \\
\text { la Innovación } \\
\text { Bankimer, 2010) }\end{array}$ & $\begin{array}{l}\text { a) la innovación planificada y aplicada a los modelos de } \\
\text { negocio y productos; b) la habilidad de las empresas privadas } \\
\text { para resolver de manera más efectiva que los gobiernos, } \\
\text { los problemas sociales y medioambientales; c) la innovación } \\
\text { colaborativa como ecosistema del futuro para poner en común } \\
\text { las ideas de una mayor variedad de agentes en un entorno } \\
\text { abierto a la participación; y d) la función de la tecnología } \\
\text { (comunicaciones, Internet) para facilitar la innovación } \\
\text { colaborativa }\end{array}$ \\
\hline
\end{tabular}




\begin{tabular}{|l|l|}
\hline Propuesta & Características \\
\hline $\begin{array}{l}\text { Harris y Albury } \\
(2009)\end{array}$ & $\begin{array}{l}\text { La innovación social está orientada por el bien social, público y } \\
\text { necesidades sociales mal servidas u obviadas por el mercado o } \\
\text { el estado y provista por el sector público, privado o de terceros } \\
\text { y necesariamente deben abordar los principales desafíos } \\
\text { sociales. }\end{array}$ \\
\hline Westall (2007) & $\begin{array}{l}\text { Centrada en objetivos sociales, productos de calidad, nuevos } \\
\text { métodos } \\
\text { de organización y/o de producción, gobernabilidad, nuevas } \\
\text { relaciones de mercado y nuevas formas jurídicas }\end{array}$ \\
\hline NESTA (2008) & $\begin{array}{l}\text { Nuevos productos, servicios o modelos para cumplir con } \\
\text { necesidades básicas insatisfechas. Puede ser transmitida } \\
\text { a través de una profesión o sector (educación o salud) o } \\
\text { geográficamente de un lugar a otro. }\end{array}$ \\
\hline OCDE (2010) & $\begin{array}{l}\text { Satisfacer problemas sociales no provistas por el mercado a } \\
\text { través de productos y servicios, procesos de integración laboral } \\
\text { y formas de participación. Se trata del bienestar de individuos, } \\
\text { comunidades, consumidores y productores }\end{array}$ \\
\hline
\end{tabular}

Fuente: elaboración propia sobre la base de lo propuesto por los autores citados.

Como se observa, los estudiosos coinciden en la mayoría de los aspectos que involucra una innovación social, quedando claro el consenso en cuanto a la atención de las necesidades sociales y ambientales no cubiertas por gobierno o el mercado y su contribución a mejorar la calidad de vida de los grupos más vulnerables. Se trata de innovaciones que solucionan problemas sociales de forma novedosa, eficaz, eficiente, sostenible, participativa e incluso, cocreativa, lo cual está en sintonía con la filosofía del deber-ser socialmente responsable. Sin embargo, también se observa en estos enfoques posturas opuestas. En un extremo, los que consideran que sólo es innovación social aquella que plantea únicamente beneficios para la sociedad y excluye el beneficio económico para la empresa. En el otro extremo, están las posturas que plantean la generación de valor económico tanto para la sociedad como para el productor. En medio, los planteamientos que ven a la innovación social como un proceso de evolución o que simplemente consideran que no hay problema social que no implique un valor económico. 
De cualquier forma, el valor que aporta el comportamiento socialmente responsable, en este caso de las empresas que generan verdaderas innovaciones sociales, quieran o no, repercutirán en su imagen y reputación y, por tanto, en su posicionamiento en el mercado, es decir, por añadidura, preferencia al momento de la decisión de compra de productos y servicios.

En esencia, resaltan como aspectos reiterativos en estos enfoques, la atención a necesidades sociales de grupos vulnerables que no son eficaz ni eficientemente atendidos por gobiernos o el mercado. Implica la sinergia que puede producirse entre el sector público, el privado y sin fines de lucro. El escenario ideal es producir cambios en valores, actitudes y procesos, entre otros, que contribuyan a un mayor bienestar de individuos, comunidades, consumidores y productores.

Ante la complejidad de los problemas de una sociedad, la innovación puede ser una opción para mejorar la calidad de vida de las comunidades a cualquier nivel: salud, seguridad, transporte, comunicaciones, seguridad en el trabajo y medio ambiente, entre otros.

\section{RSE Y LA FUNCIÓN DE LA INNOVACIÓN}

La vinculación entre RSE e innovación implica comprender la naturaleza de la orientación socialmente responsable y cómo se imbrica en este contexto el accionar innovador. En este sentido, se presenta un breve recorrido teórico sobre RSE e iniciativas que apuntan a la innovación como acción socialmente responsable.

\section{RSE, INNOVACIÓN Y SU PROCESO EVOLUTIVO}

Existen múltiples conceptos sobre RSE entre los cuales es propicio citar el planteado recientemente por la Comunidad Europea (2011c:7), como la "responsabilidad de las empresas por su impacto en la sociedad" que implica como requisito previo el respeto a la ley y los convenios colectivos. En este contexto, la empresa y los grupos de interés deben integrar las preocupaciones sociales, medioambientales, éticas, de derechos humanos y de los consumidores en sus operaciones empresariales con el fin de maximizar la creación de 
valor compartido entre estos grupos e identificar, prevenir y atenuar las consecuencias adversas de sus actividades.

Por su parte, Guedez (2006) propone tres dimensiones del concepto. Una definición connotativa que la define como el ejercicio ético y sustentable de la competitividad; otra descriptiva, en la cual RSE es un conjunto de estrategias que permiten identificar y atender, anticipar y sobrepasar, las necesidades, expectativas y capacidades de los grupos de interés internos y externos; y una tercera, operativa, que complementa los conceptos anteriores y pone de manifiesto su operacionalización.

En cuanto a los ámbitos de acción de la RSE, la Comisión Europea (2001), en su Libro Verde la concibe en dos dimensiones, la que aborda la gestión del recurso humano, la salud y la seguridad del puesto de trabajo, la gestión del impacto ambiental y de los recursos naturales, recogidos en la dimensión interna; y la referida a las comunidades locales, los socios comerciales, los proveedores y los consumidores, derechos humanos, problemas ecológicos y desarrollo sostenible, incluidos en la dimensión externa. En esencia, la RSE orienta las acciones empresariales para lograr la protección del entorno natural y contribuir a mejorar la calidad de vida de empleados, sus familias, los clientes y demás grupos; paralelamente se estará contribuyendo con el desarrollo económico de las sociedades.

De igual forma, las organizaciones deben entender que su sostenibilidad y la de su entorno, pasa por considerar no sólo los criterios económicos, sino también los sociales y medioambientales, es decir, considerar el concepto de la triple economía. En este orden de ideas, debe considerarse los resultados a largo plazo (económica), la minimización del impacto de las operaciones y los productos de la empresa, incluso, la compensación de los daños ocasionados en el pasado (medio ambiente) y el fomento del bienestar de los grupos de interés, como mínimo, afectados en el ámbito en el que actúa (social).

Asimismo, la RSE puede verse como un camino evolutivo, en el cual las empresas se inician con acciones sociales quizás no imbricadas en su gestión estratégica, pero que pueden considerarse pasos adelante en esta orientación. El modelo planteado por Guedez (2008) puede ilustrar esta evolución. Se encuentra en primera instancia 
la organización económicamente responsable, es decir, se busca garantizar la rentabilidad y sostenibilidad en el tiempo, por supuesto, en un ambiente de legalidad. La siguiente etapa es la consideración de la organización como públicamente responsable, es decir que tal responsabilidad pueda ser percibida en el desarrollo o prestación de productos y/o servicios de calidad, precio justo, empleos dignos y cumplimiento de la ley, entre otros. Lo que importa en esta etapa es "cuánto gano con lo que hago" y se trata generalmente de filantropía y proyectos de RSE.

La organización socialmente dispuesta es la empresa convencida, consciente, que decide hacer algo al respecto y busca orientación a su decisión; deja emerger su conciencia social reconociendo su función dentro de la sociedad y cómo ésta le provee las condiciones necesarias para permanecer y crecer. A este nivel de madurez y conciencia, se generan estrategias sociales que buscan compensar a la sociedad y lo ideal sería que tal forma de pensar emane también de la dirección. En la organización socialmente competente, además de la conciencia y decisiones enmarcadas en lo socialmente responsable, cobra importancia la apropiación de competencias, es decir, información, conocimiento, habilidades, destrezas, actitudes y valores asociados a las exigencias del negocio. La responsabilidad social en sí misma es una competencia que implica capacidad, disposición (creencia, conocimiento y sensibilidad) de los miembros de una organización para orientar su compromiso social. La competitividad de las organizaciones se fundamenta en el desarrollo de las competencias individuales y grupales. Es posible que las competencias individuales no garanticen las competencias organizacionales, pero no obstante, no pueden alcanzarse integralmente si se obvian las competencias de sus participantes. A este nivel lo que importa es "cuánto crezco con lo que hago", y se materializa a través de programas y estrategias de RSE.

La empresa sigue evolucionando y emerge la organización socialmente inteligente, la cual integra la disposición, la competencia y además, es capaz de institucionalizar estructuralmente en la empresa, a través de un trabajo en equipo, su responsabilidad social (misión, visión, procedimientos, programas de información, formación, cultura, iniciativas, políticas de rendición y balance social). No 
se desgasta en lograr el equilibrio, sino en ampliar la capacidad de equilibrar, no importa las condiciones y coyunturas del sistema en el cual se inserta la organización. Por último, con la mayor conciencia social está la organización socialmente ética, es decir una empresa cuya responsabilidad económica es reconocida públicamente por el cumplimiento de estándares de calidad, muestra disposición, capacidad, inteligencia organizacional y que considera al ser humano como el eje central del sistema; se fundamenta en el respeto a los derechos humanos, el fomento del capital social y de la sostenibilidad. Ahora, lo que importa es "cuánto aporto con lo que hago", e implica entonces a la RSE como modelo de gestión.

Sin duda, todos estos cambios evolutivos implican una clara orientación innovadora, en la cual el talante social asume un papel protagónico. En las diferentes etapas, tiene una función protagónica en el accionar socialmente responsable, una especial forma de innovación: la innovación social.

Para Llano (2004), "el nombre actual de responsabilidad social es innovación” como la única forma de cumplir la misión, ser competente y competitivo y este cometido se logra a través del ejercicio de la inteligencia, buscando formas de pensar abiertas, es decir, "desmarcarse de los principios vigentes y pensar desde la misma realidad con actitudes inconformistas y radicales" (por supuesto sin caer en la anarquía). El esfuerzo creativo requiere espontaneidad y capacidad de prever el orden de lo nuevo y adaptarse a los cambios, en este caso, adaptarse creativamente orientando las innovaciones sociales en un marco de RSE. Es tal la importancia de la innovación en el marco de la RSE, que importantes organismos internacionales dedican esfuerzos a impulsar esta actividad. A continuación se reseñan algunas de estas iniciativas.

\section{INICIATIVAS INSTITUCIONALES DE RSE RELACIONADAS CON LA INNOVACIÓN}

La importancia otorgada actualmente a la RSE, se debe, en parte, a la atención y esfuerzo ofrecido por los organismos internacionales reconocidos. En este sentido, se detallan:

Comunidad europea. Desde el marco de la comunidad, se tienen diferentes iniciativas. Se puede citar el Libro Verde de la Comisión 
Europea (2001), en el cual se plantea la necesidad de innovaciones tecnológicas que conlleven la disminución del consumo de recursos, la minimización de la producción de desechos y emisión de agentes contaminantes, para evitar así, el daño al entorno natural y los gastos de descontaminación. En la sección dedicada a la gestión del impacto ambiental y de los recursos naturales, plantea lo ventajoso de reducir los gastos energéticos, a la vez que se cuida el ambiente y mejora la rentabilidad y competitividad. Las empresas deben ser responsables de la contaminación de los espacios y recursos naturales, pero además, esta responsabilidad debe ser enmarcada en todas las actividades de la cadena de producción y consumo.

En otras palabras, la responsabilidad debe ser planteada en colaboración de sus socios comerciales, proveedores, distribuidores y comunidad, orientando las inversiones en investigación y desarrollo que fomenten la invención e innovación de productos, servicios y procesos que permitan una producción limpia que favorezca a la empresa, comunidad y el ambiente. Incluso, se plantea la pertinencia de que las grandes empresas faciliten la expansión de pequeñas empresas innovadoras que luego, a través del intercambio comercial, contribuirán con la cadena de valor de la empresa que la fomenta, en una relación de ganar-ganar. Desde el punto de vista de los consumidores, éstos exigen productos socialmente responsables que no perjudiquen el ambiente, lo que amerita cambios en el diseño de productos y servicios, así como de sus procesos de producción.

Asimismo, la Comisión Europea $\left(2011^{\mathrm{b}}\right)$ plantea otras iniciativas tales como el "Estado de la Unión por la Innovación 2011". Esta resalta la apuesta por la innovación social como elemento clave en la solución de problemas claves de la región y "Empowering people, driving chance. Social Innovation in the European Union”, que promueve que la sociedad civil y los empresarios sociales participen creativamente con iniciativas dirigidas a los grupos vulnerables y respondan a las crecientes necesidades sociales no suplidas por el mercado o los gobiernos. Estas iniciativas contribuyen a través de la innovación, particularmente social, al campo de la responsabilidad social.

Pacto mundial. Propuesto en 1999 por el Secretario General de las Naciones Unidas, Kofi Annan, y firmado en el Foro Económico Mundial (Suiza) entre el empresariado mundialy las Naciones Unidas, 
está basado en el cumplimiento de diez principios que deberían adoptar las empresas y que posteriormente han sido retomados por otras iniciativas. Los mencionados principios incluyen aspectos de derechos humanos, laborales, ambientales y anticorrupción, ante lo cual las empresas deben: apoyar y respetar la protección de los derechos humanos fundamentales, asegurarse de no ser cómplices en la vulneración de los derechos humanos, apoyar la libertad de asociación y el reconocimiento eficaz del derecho a la negociación colectiva, la eliminación de toda forma de trabajo forzoso o realizado bajo coacción, la erradicación del trabajo infantil, la abolición de las prácticas de discriminación en el empleo y la ocupación, favorecer la adopción del enfoque de prevención frente a las necesidades medioambientales, fomentar iniciativas que promuevan una mayor responsabilidad ambiental, fomentar el desarrollo y difusión de tecnologías respetuosas con el medio ambiente y trabajar contra la corrupción en todas sus formas, incluidas extorsión y soborno (Red del Pacto Mundial, http://www.pactomundial.org).

Según el pacto, las empresas deben considerar que el daño causado al ambiente conlleva el deterioro de la imagen de la empresa, lo cual es mucho más caro que las posibles inversiones para evitar el daño al ambiente.

Naciones Unidas, Agenda 21. En el contexto del Pacto Mundial nace la Agenda 21, la cual comparte y expone claramente la necesidad de innovación en el fortalecimiento del comercio, la industria, incluyendo las empresas transnacionales, que deben buscar mayor eficiencia a través de procesos de producción, estrategias preventivas, tecnologías y procedimientos de producción limpia (en cada una de las etapas del ciclo de vida del producto) a los efectos de reducir o evitar desechos que perjudiquen el medio ambiente y fomentar así el desarrollo sostenible. Las innovaciones tecnológicas, el desarrollo, las aplicaciones, la transferencia de tecnologías y los aspectos más generales de la asociación y la cooperación son cuestiones que en gran medida incumben al comercio y la industria. La Agenda 21 propone dos programas en este sentido: uno dirigido al fomento de la producción limpia que utilice la innovación en tecnología y gestión de recursos de manera eficaz, minimizando desperdicios perjudiciales para el ser humano y el ambiente y fabricando productos que puedan 
ser reciclados; así como también, un programa dirigido al fomento de la responsabilidad social (Organización de las Naciones Unidas, http://www.un.org/esa/sustdev/documents/agenda21/spanish/agenda 21sptoc.htm).

Red Iberoamericana de Indicadores de Ciencia y Tecnología (RICYT, 2001). La RICYT, a través del Manual de Bogotá, dedica un apartado a la innovación como proceso social e interactivo y destaca la importancia de establecer canales de comunicación a largo plazo entre la empresa y sus grupos de interés. A su vez, plantea la difusión de innovaciones entre los agentes económicos aprovechando la retroalimentación que permite este intercambio. Las relaciones entre empresas u otros agentes (proveedores, distribuidores, contratistas, instituciones) dan lugar a vínculos de cooperación y confianza que permiten obtener y beneficiarse de información tecnológica y de mercados y detectar oportunidades. Por supuesto, esto tendrá una orientación socialmente responsable, en la medida en que estos vínculos promuevan el desarrollo de iniciativas empresariales locales que a través del apoyo innovador de sus aliados, impulsen el desarrollo regional. Asimismo, la RICYT, aborda el tema medioambiental y propone en el marco de los objetivos asociados a la calidad, los relacionados a las condiciones de trabajo y la disminución del impacto ambiental (indicadores de gestión ambiental: impacto ambiental de las innovaciones de producto, proceso y organizacionales en aguas, atmósfera, suelos, paisaje, consumo de energía y residuos, productos ambientalmente sanos y producción más limpia, entre otros).

Lo planteado hasta ahora permite comprender que el camino hacia una empresa en verdad socialmente responsable, o según Guedez, "socialmente ética", es un proceso evolutivo que implica varias etapas. Por supuesto, el ideal es que la RSE debe ser más que filantropía o iniciativas sociales o de mercadeo aisladas de la gestión estratégica de la empresa, pero si se consideran las etapas más tempranas de madurez socialmente responsable de las empresas, es común encontrar acciones de este tipo. Como puede intuirse, la orientación de la RSE está todavía incubándose en el empresariado y de allí el esfuerzo permanente de los organismos internacionales, creación de marcos legales y normativos para estimular y propulsar su cumplimiento. En este sentido, 
...expertos del Future Trends Forum coinciden en que todavía queda por definir, estandarizar y compatibilizar el concepto de la RSE y su aplicación. Pese al gran progreso alcanzado en este ámbito, todavía queda mucho camino por recorrer a la hora de asociar las iniciativas socialmente responsables a la estrategia corporativa y de emplear la innovación como medio para desarrollarlas. (Fundación de la innovación Bankinter, 2009: 6)

Al ser un proceso de aprendizaje evolutivo es común que en etapas tempranas existan proyectos o programas de responsabilidad social que disten todavía del deber ser promulgado por la RSE, pero que pueden ser considerados como innovaciones sociales dignas de resaltarse. No obstante, debe reiterarse que el escenario ideal es que estas empresas sigan avanzando para convertirse en una empresa "socialmente ética" que considere al ser humano como eje central del sistema y se fundamente en el respecto a los derechos humanos, fomente el capital social y la sostenibilidad.

\section{Experiencias de innovación social en el contexto de la RSE en Venezuela}

Tal como se ha mostrado en el recorrido teórico, es posible encontrar diferentes puntos de vista sobre lo que debe considerarse como innovación social. No obstante, para el cometido de este estudio, se considerarán los puntos de convergencia de los autores como criterios para considerar la acción empresarial como socialmente innovadora. Asimismo, no se formulará ningún juicio con respecto a la etapa socialmente responsable en que se encuentre la empresa, pues esto amerita un estudio diferente del aquí planteado que por demás es interesante y pertinente. Visto así, podrán considerarse innovadoras en el marco de la RSE, las acciones que puedan estarse desarrollando en etapas tempranas en la orientación socialmente responsable (filantropía, programas y voluntariado, entre otros).

En el escenario venezolano, son múltiples y de naturaleza variada las iniciativas innovadoras que han resultado de la adopción de criterios socialmente responsables, iniciativas, por demás exitosas, muchas en escenarios de participación y diálogo social. El interés es mostrar casos en diferentes sectores tales como alimentos y bebidas, 
banca telecomunicaciones y empresas del Estado. En este sentido, puede considerarse la actividad como innovadora socialmente si responde a una necesidad social o ambiental no cubierta por el gobierno o mercado y contribuye a mejorar la calidad de vida de los grupos vulnerables de forma novedosa, eficiente, sostenible, participativa e incluso, cocreativa ${ }^{3}$.

Así, en el sector alimentos, es interesante abordar la empresa venezolana más importante del país, Empresas Polar, la cual a través de la Fundación Polar orienta sus actividades a la integración e innovación en prácticas de compromiso social sustentables con sus grupos relacionados. Hace hincapié en la incorporación de novedosas herramientas de gestión, así como en la medición de los avances y progresos, alineados además, con el cumplimiento de los Objetivos del Milenio de las Naciones Unidas. Su gestión social busca innovar en tres grandes campos: educación, salud y desarrollo comunitario (http://www.fundacionempresaspolar.org/\#/home).

Entre otras, podría resaltarse como iniciativas sociales innovadoras, las referidas al "Programa de capacitación para el trabajo y el emprendimiento" y, específicamente, la acción relacionada con la capacitación femenina para el trabajo por medio de formación y apoyo a iniciativas para el desarrollo de competencias y destrezas en las mujeres. Asimismo, es plausible el "Fortalecimiento de Unidades de Gestión Económica Local” que provee asesoría y acciones para el fortalecimiento de cooperativas locales proveedoras de alimentos preparados y adecuación a las mejores prácticas de fabricación (manipulación de alimentos). También, puede considerarse el "Centro Nacional para Pequeños Productores Agropecuarios”, a través del cual se ofrece capacitación para la gestión de sistemas sostenibles de producción agropecuaria y técnicas de campo, pasantías y asistencia, entre otros.

\footnotetext{
${ }^{3}$ Debe aclararse que aún cuando se menciona grandes y medianas empresas del país, de ningún modo se excluye que la pequeña empresa sea proclive a generar innovaciones sociales en sus procesos y en sus relaciones con los grupos de interés. La elección de los casos estudiados responde más a un tema de practicidad por el acceso a la información a través de las páginas Web de estas empresas, que a la consideración de la innovación como exclusiva de este tipo de empresa. Por supuesto, no se desdeña en futuras investigaciones el abordaje de la innovación social de las pequeñas empresas locales.
} 
Se puede mencionar la contribución de Polar a la cadena agroproductiva del cacao, en la que participa la Asociación Civil Chocolates La Flor de Birongo y la Cooperativa Cocobí (sector Los García del municipio Brión, estado Miranda), la cual se encarga de la fermentación del cacao y asegurar así la materia prima que requiere la Asociación Civil La Flor de Birongo. Se encuentra también el apoyo a la Cadena Agroproductiva del Bambú y el apoyo a organizaciones artesanales que aprovechan este recurso y benefician a la comunidad. Agricultores, artesanos y sus familias, se ven beneficiados con la elaboración de chocolates y mermeladas y productos elaborados con bambú que generan ingresos a las familias y progreso a la región. Destacan también, las cadenas agroproductivas del maíz, las cuales concentran el trabajo de familias campesinas del estado Yaracuy que trabajan en pequeñas parcelas, propiciando la creación de cooperativas.

Sin duda, el impacto que representa el abordaje social de la comunidad a través del empoderamiento de la mujer como eje motor de la sociedad y la economía, así como el apoyo al emprendimiento, microempresario y a la agroindustria, requiere de proyectos innovadores cuyos resultados se aprecian en la contribución al desarrollo endógeno de las regiones que impacta, las familias y mejora la calidad de vida. El abordaje de las comunidades lo realizan a través de estudios diagnósticos que orientan las iniciativas que realizan y que retroalimentan su constante acercamiento comunitario. Al generar fuentes de trabajo, mayores ingresos para las familias, productos sustentados en tecnologías limpias, formación, integración e inclusión puede considerarse en general que se trata de innovaciones sociales.

De igual forma, en el sector de alimentos y bebidas se encuentra la Fundación Santa Teresa (C.A. Ron Santa Teresa), cuyas acciones innovadoras desarrollan proyectos auto-sustentables de responsabilidad social para garantizar el crecimiento del municipio Revenga (Aragua) en áreas como seguridad, educación, salud, capacitación para el trabajo, promoción y sensibilización turística y cultura. A través de programas como Visión Revenga, se busca transformar a Revenga en un modelo próspero y con calidad de vida. En este proyecto, los integrantes de comunidad par- 
ticipan para definir el plan estratégico en temas como seguridad, vivienda, turismo, formación para el trabajo y valores. Es relevante también el Proyecto Alcatraz, creado en el 2003, que tiene como fin erradicar la delincuencia y transformar el liderazgo violento de jóvenes transgresores en liderazgo virtuoso. Se reclutan jóvenes con problemas de conducta de Revenga quienes participan de un programa que incluye trabajo intensivo, formación en valores, práctica de rugby, asistencia psicológica y trabajo comunitario. En el contexto de este accionar innovador, en 2005 se creó la Red de Madres Alcatraz para promover el desarrollo integral de la mujer. Las madres son formadas en valores, adquieren destrezas y mejoran su autoestima para generar responsabilidad y compromiso que coadyuven en el proceso de reinserción social de sus familiares (http://www.fundacionsantateresa.org/fundacion. php?lang=esp).

En estos proyectos, se puede ver la atención a necesidades sociales de diferente naturaleza. Se han generado alianzas con entes gubernamentales y de la sociedad civil (líderes comunitarios, consejos comunales, asociaciones culturales y deportivas, autoridades municipales, cuerpos de seguridad y empresas, entre otros) y metodologías participativas que son claros ejemplos de inclusión y participación. Los participantes de estos procesos contribuyen con ideas, financiamiento y apoyo institucional en el logro de este cometido y los proyectos han mostrado permanencia en el tiempo. Asimismo, es una experiencia que demuestra formas verdaderamente novedosas y eficaces de responden a problemas sociales.

De igual manera, regenerar jóvenes con conductas transgresoras y formarlos profesionalmente, incluyendo a sus familias, es un ejemplo de innovación social digna de resaltar. Se trata de jóvenes que rechazados por su pasado antisocial (que incluso han afectado a la empresa y sus empleados) y que, sin embargo, la empresa los ha apoyado y ha asumido el riesgo de reformarlos e involucrarlos a la vida productiva de la empresa y la región. En la visión de valor compartido, es una demostración de que puede generarse valor tanto para la comunidad como para la empresa, pues esta última garantiza un recurso humano formado, seguridad para las instalaciones y empleados y la comunidad se beneficia del desarrollo local. Es significativo 
el proceso participativo a todos los niveles del Municipio que se origina en los proyectos de la fundación.

Coca-Cola FEMSA sustenta su comportamiento social a través de cuatro ejes: calidad de vida en la empresa, salud y bienestar, vinculación con la comunidad y cuidado del medio ambiente. Resalta el eje de salud y bienestar bajo el lema "Promovemos el deporte y decimos NO a las drogas" y el programa Vamo' a jugá', que busca fomentar el deporte en niños con escasos recursos de todo el país. Sin duda, mantener alejados a los niños y jóvenes del flagelo de la droga es una necesidad social imperante, sobre todo, en los sectores más pobres. Mediante estos programas, la empresa busca involucrar a los niños y jóvenes con el deporte. En este caso, se beneficia directamente a las comunidades al contribuir con la inclusión de la población menos favorecida y con la disminución de los índices de deserción estudiantil y delincuencia.

En cuanto al eje de cuidado del medio ambiente, a nivel de Latinoamérica, Coca-Cola FEMSA ha invertido en tecnologías que permiten tener empaques amigables con el medio ambiente. Se han optimizado tapas, etiquetas y material de empaque para transporte de producto. No hay que profundizar mucho para entender el grave problema que significa el cambio climático, ante lo cual la iniciativa social de Coca-Cola es un ejemplo de conciencia y respeto ambiental. Más allá de iniciativas puntuales, se esfuerzan por llevar su alcance innovador a su cadena de valor y, específicamente, con la utilización de la producción limpia apuesta por tecnología que minimice el impacto de sus operaciones en el medio ambiente.

En otro ámbito de acción, sin duda, una de las más recientes innovaciones sociales es la representada por los negocios inclusivos, es decir la atención de las necesidades de los consumidores de la base de la pirámide o sectores de bajos ingresos. Se considera como innovación social, pues satisfacen necesidades sociales, generan valor a la sociedad, implican alianzas público-privadas-sin fines de lucro en una experiencia de co-creación, participan las personas relacionadas, son sostenibles e incluso rentables. No sólo se considera el sector como potencial mercado de consumidores, sino que, más allá de servirlos, busca la co-creación de negocios inclusivos en los que 
participen y formen parte de la cadena de valor, ya sea como proveedores, distribuidores o consumidores ${ }^{4}$.

Según el Future Trends Forum, reseñado por la Fundación para la innovación Bankimer (2009), ofrecer productos y servicios a más 4.000 millones de consumidores que viven con menos de dos euros al día, puede ser la base de la nueva economía global y fuente de generación de nuevas innovaciones sociales que redunde en beneficios para todos. Es un ejercicio de participación entre empresas, organizaciones sin fines de lucro, gobiernos locales y sociedad civil, es decir, verdaderas innovaciones sociales que dan lugar a nuevos empresarios, nuevos consumidores, desarrollo económico, inclusión y transformación social. En esencia, reducir la pobreza generando riqueza (Prahalad, 2006) ${ }^{5}$.

Un ejemplo de negocios inclusivos es el sector farmacéutico y, específicamente, las Farmacias SAAS y el modelo de negocio de las "botiquerías populares": la farmacia de la cuadra. Es un caso de negocio inclusivo de co-creación, en el cual pequeños locales de sectores populares que adolecen de este servicio, son utilizados para

\footnotetext{
${ }^{4}$ La estrategia de inclusión llega a su máximo alcance cuando las empresas, en lugar de construir una estructura organizacional basada en sus fortalezas, desarrollan esquemas híbridos de funcionamiento flexible con líderes comunitarios, microempresarios de las comunidades o personas de escasos recursos que se convierten en proveedores, distribuidores o vendedores. Para ello, las empresas invierten en planes de creación de empleos productivos en zonas vulnerables y en capacitación para desarrollar la identificación con el negocio entre los nuevos aliados (Culshaw, 2010: 33).

${ }^{5}$ En el contexto del sector de bajos ingresos, se observan los modelos pay-peruse que permite a los consumidores pagar precios más bajos por el uso de un producto, servicio o instalación, en lugar de desembolsar una cantidad elevado; el modelo no frills service que cubre las necesidades de los más pobres a precios muy bajos gracias a un recorte en los extras, pero manteniendo la calidad produce beneficios por alto volumen generado por el elevado uso de los activos y la especialización del servicio. El modelo paraskilling complementa al anterior y desagrega procesos de negocio en tareas más simples que puedan realizar trabajadores con escasa cualificación. También, plantean modelos con nuevos canales de distribución para llegar a zonas rurales (Future Trends Forum, en Fundación para la Innovación Bankimer, 2009). Otros ejemplos de negocios inclusivos pueden consultarse en Brugmann y Phahalad (2007), Prahalad (2005), Fundación de la innovación Bankinter (2009) o Culshaw (2010).
} 
la venta de medicinas, productos de cuidado personal y alimentos básicos a precios convenientes y cerca del consumidor. Con una pequeña inversión los recién graduados farmacéuticos se incorporan al mercado de las mayorías como parte de la cadena de valor. Se genera empleo para aprendices o auxiliares que provienen del barrio que son capacitados en el oficio, llegando incluso a generar sus propias empresas familiares (Culshaw, 2010).

Otro ejemplo de negocio inclusivo en el sector farmacéutico es Pfizer Venezuela, a través de su programa "Comunidad más saludable" de distribución y venta de medicinas a precios asequibles en barrios populares, pero aprovechando a sus habitantes como sus representantes para lo cual los contrata y forma. Los representantes son parte de la comunidad y pasan a formar parte de la cadena de valor de la empresa. De esta forma tiene acceso a este mercado y, simultáneamente, se genera empleo, progreso y calidad de vida (Culshaw, 2010).

En estos casos del sector farmacéutico, se trata de sectores populares que no tienen acceso a estos productos y servicios y que a través de una estrategia de valor compartido, se generan alianzas que contribuyen con valor para ambas partes. Sólo basta imaginar el sacrificio que representa para los habitantes, por ejemplo, de las barriadas caraqueñas tener que desplazarse escaleras arriba y abajo para adquirir los productos más básicos para una familia y sin dejar de contar el peso que representan en su presupuesto. Esta población excluida es ahora considerada un modelo de negocio que satisface sus necesidades a precios bajos, reduce costos de desplazamiento (utilidad de lugar y tiempo) y que además los involucra y les permite generar ingresos y bienestar. Simultáneamente, es una forma innovadora de ampliar la red de distribución en una acción de ganarganar en lugares antes no atendidos.

En otro contexto, la banca se ha unido a la experiencia de las microfinanzas y surge la banca comunitaria en la cual resalta el apoyo a emprendedores y la oportunidad para los microempresarios de acceder a financiamiento. Por ejemplo, la Banca Comunitaria Banesco que, además de microcréditos, provee a los microempresarios educación financiera, cursos sobre desarrollo de negocios y contrata a personas de los barrios que demuestran compromiso (Banesco, 
2011). Es una forma de contribución al desarrollo local de la región, al emprendimiento, disminución de pobreza y en general, a la innovación social ${ }^{6}$.

El Banco Venezuela y el Grupo Santander ofrecen programas educativos tales como Universia, Programas Universidades y programas culturales. Además, ofrecen iniciativas como la cybercuenta universitaria, carnet inteligente, becas trabajo, becas estudio y financiamiento de proyectos tecnológicos (http://www.bancodevenezuela.com /?bdv=link_qsomos\&id=172). El Banco Mercantil, a nivel de educación superior, apoya a través del Fondo de Capital Social Mercantil programas de financiamiento de becas; equipamiento de laboratorios y salas de computación, investigación y desarrollo y creación de cátedras fundacionales (Banco Mercantil, 2011).

Ante la situación que enfrentan las universidades de falta de recursos, existen necesidades todavía pendientes con las cuales, sin duda, se contribuye con estas iniciativas. De una u otra forma, estos programas ayudan a disminuir la deserción universitaria y son una posibilidad para la educación e investigación. Son proveedores de desarrollo para los estudiantes menos favorecidos económicamente y para sus comunidades, y contribuyen a mejorar el sistema educativo a través de la inversión social en las instituciones universitarias.

En el sector de telecomunicaciones, Telefónica Venezuela ofrece el programa Educared, que plantea mejorar la calidad de la educación y fomentar la igualdad de oportunidades mediante la aplicación de las nuevas tecnologías de la información y la comunicación a los procesos de enseñanza y aprendizaje, desarrolla investigación global avanzada sobre el análisis y el conocimiento de las tecnologías de la información y las comunicaciones y su impacto en la sociedad. Adicionalmente tienen el programa Proniño, orientado a la prevención y progresiva erradicación del trabajo infantil a través de la escolarización de niños, niñas y adolescentes de los estratos socioeconómicos más desfavorecidos (http://www.fundacion.telefonica. com.ve). En este caso se contribuye con educación a disminuir la

${ }^{6}$ La banca comunitaria de Banesco resultó ganadora del Programa de Tecnologías para la Inclusión Financiera organizado por el Banco Interamericano de Desarrollo, el Fondo Multilateral de Inversiones y la Corporación Andina de Fomento (Banesco, 2011). 
brecha tecnológica y el análisis constante en materia de tecnologías e información y comunicaciones, que propicia la creación de proyectos innovadores que impacten en el progreso de la sociedad y en el aprovechamiento de este recurso para la generación de redes de aprendizaje y transferencia. La atención del infante y la garantía de sus derechos son base fundamental en la generación del ciudadano del futuro. Con estas iniciativas innovadoras se contribuye al desarrollo económico, social y cultural fomentando la igualdad, inclusión, educación y menos pobreza.

Una mirada al sector turismo, pone en relieve a la Fundación Programa Andes Tropicales. Esta fundación sin fines de lucro ha creado las redes de turismo de base comunitaria entre los parques nacionales Sierra Nevada y la Culata estado Mérida. El programa ofrece una alternativa turística sencilla con rutas de excursionismo unidas por mucuposadas, es decir, casas de los habitantes del poblado que son recuperadas para ofrecer servicios de alojamiento y comida por parte de sus pobladores (http://www.andestropicales. org/front_andes_vzla.html).

Esta iniciativa es un ejemplo de innovación social que se ha mantenido y crecido con el tiempo. Ha desarrollado una metodología sui generis de trabajo para instaurar en algunos pueblos un modelo de negocio que beneficia a la comunidad. Involucra levantamiento cartográfico, diseño de rutas, capacitación de servicios, asesoramiento, supervisión y restauración de viviendas (mucuposadas), entre otros. Los propietarios de las mucuposadas pasan a ser micro-empresarios y personas claves en este modelo de convivencia sustentable; generan ingresos para sus familias y progreso para la población. Un modelo admirable que respeta y consolida la cultura de los pueblos y su relación con el medio ambiente.

Desde de la industria petrolera del país, también se realizan continuamente inversiones sociales en apoyo a la política pública social del gobierno venezolano a través de Petróleos de Venezuela, Sociedad Anónima (PDVSA). Así, las "misiones sociales" como estrategias masivas orientadas a garantizar los derechos fundamentales a la población, con énfasis en los sectores más excluidos, reciben de PDVSA recursos, planificación y coordinación. PDVSA apoya directamente a casi todas las misiones sociales y programas de desarrollo impulsadas 
actualmente en el país. La empresa del estado, PDVSA, ha tenido que abordar los problemas sociales que aquejaban por una u otra causa a diferentes grupos excluidos y con necesidades básicas no cubiertas (PDVSA, 2009).

Puede reseñarse la misión Robinson I y II que abordó la población analfabeta del país y proveyó los recursos humanos y técnicos necesarios para que lograran culminar la educación primaria. Los resultados permitieron minimizar los índices de analfabetismo del país en dos años (1.482.000 venezolanos) y Venezuela fue declarada "territorio libre de analfabetismo" el 28 de octubre de 2005. La misión Guaicaipuro vino a responder a una demanda histórica de la sociedad al reconocer la existencia de los pueblos y comunidades indígenas. Otras misiones que apuestan a la inclusión de grupos menos favorecidos y que han demostrado su eficiencia y puesto en alto el nombre del país, es la misión Música a través de la consolidación del sistema nacional de orquestas y coros juveniles e infantiles de Venezuela

En definitiva, todas las iniciativas descritas, de una u otra forma, vienen a contribuir con la diminución de los grandes flagelos de nuestra sociedad latinoamericana: la pobreza y la desigualdad que a la postre mantienen necesidades básicas aún no resueltas. Se han considerado, pues cumplen con características vinculadas a la innovación social propuestas por los estudiosos del tema. Sin duda, son pasos adelante en función de alcanzar a ser una verdadera empresa socialmente responsable que incluya en su pensamiento y gestión estratégica a la RSE.

\section{Conclusiones}

La RSE plantea cambios importantes sobre los cuales las empresas deben reflexionar, colaborando con el desarrollo de nuevas realidades que fomenten el bienestar de su entorno social. Esto no sólo implica vender más productos o servicios en una búsqueda de rentabilidad, al contrario, exige dar supremacía a la dignidad del ser humano y al medio ambiente, y alrededor de esta premisa, satisfacer sus necesidades. La realidad exige un cambio de acción por parte de las empresas, tanto desde el enfoque de la RSE como desde la innovación. 
La innovación y los sistemas que la enmarcan han sido fuente generadora de progreso para los países y no son pocas las iniciativas que propulsan su desarrollo. No obstante, aun cuando se han logrado innegables avances producto de la innovación, siguen existiendo (o se han obviado) necesidades sociales y medioambientes pendientes de satisfacer. Emerge así el interés en abordar el interés social y medioambiental a través de la innovación, lo cual deja entrever la consideración de elementos adicionales a los considerados tradicionalmente. En sintonía con esta idea, los organismos internacionales vinculados a la RSE prevén lineamientos que contribuyan al fomento de la innovación. Los manuales de Oslo, Bogotá, Libro Verde de la Comisión de las Comunidades Europeas, Pacto Mundial y Agenda 21 de las Naciones Unidas, entre otros, son muestras de ello y en sus abordajes se nota una clara prevalencia de la temática social y ambiental.

La consideración de lo socialmente innovador sigue planteando diferentes puntos de vista, no obstante, lo que sí está claro son los puntos de convergencia de los estudiosos de la materia y tienen que ver con la satisfacción de necesidades sociales no abordadas adecuadamente por gobiernos o el mercado. Deben representar una forma novedosa, sostenible y eficiente de satisfacer esta necesidad y lo ideal es que se generen en ambientes de participación e inclusión de los grupos más vulnerables de la sociedad y que provean un cambio de actitud y valores.

De igual forma, el cambio de la empresa hacia una orientación "socialmente ética" como el ideal a lograr en el marco socialmente responsable, se produce a través de un proceso de madurez por etapas que implica tiempo y aprendizaje. En este sentido, aunque no es objetivo de este estudio ubicar a la empresa en alguna etapa específica de la evolución mencionada, es posible intuir que independientemente del nivel alcanzado, se pueden encontrar innovaciones sociales que cumplan con las características mencionadas. Es decir, es posible que la empresa no haya conseguido la madurez necesaria para imbricar a la RSE en su gestión estratégica y considerar como eje central del sistema al ser humano fundamentado en el respeto a los derechos humanos, el fomento del capital social y de la sostenibilidad y que, sin embargo, generan innovación social. 
No obstante, puede encontrarse en el camino hacia su reconversión como la empresa socialmente ética que requiere nuestra sociedad y contribuir a la generación de procesos y relaciones que hagan surgir innovaciones provechosas para todas las personas involucradas en el desarrollo local, regional y nacional.

En el caso de Venezuela, existen numerosas empresas en el camino de lo socialmente ético, que dedican estudios y análisis rigurosos, tiempo y recursos a apoyar actividades que favorecen el bienestar de sectores poco favorecidos del territorio. Más allá del interés que persigan, o la etapa en que puedan estar transitando en la evolución del cambio hacia una empresa socialmente responsable, de alguna u otra forma contribuyen al beneficio de las comunidades en el orden social y ambiental mostrando una forma diferente de solucionar un problema latente en las comunidades. En tal sentido, tales iniciativas pueden ser consideradas como innovaciones sociales que apoyan el progreso de la empresa en la madurez que se requiere para consolidarse como empresas socialmente éticas y que, sin duda contribuyen con la sociedad y el ambiente en un contexto sostenible.

Por supuesto, es un reto para todos (estado, empresas, comunidades, universidades y demás integrantes que viven en esta sociedad) lograr generar alianzas, conciencia social y ambiental que impulsen la innovación con carácter social en pro de un mundo mejor. No puede desdeñarse la importancia y sinergia que puede generarse de la actuación conjunta de los sectores público, privado y sin fines de lucro. La experiencia de cada sector en su contexto favorecería en conjunto el abordaje integral y óptimo de las necesidades sociales que aún afectan a nuestras sociedades menos avanzadas. Sin duda, sería una fuente generadora de innovaciones sociales con impacto en la sociedad. 


\section{Referencias}

Alburquerque, F. (2003). Curso sobre desarrollo económico local. Curso OIT: estrategias para el desarrollo económico local. Turín: Mimeo.

Banco de Venezuela. (n.f.). Disponible en http://www.banco devenezuela.com/?bdv=link_qsomos\&id $=172$

Banco Mercantil. (n.f.). Disponible en http://www.bancomercantil. $\mathrm{com} / \mathrm{mercprod} /$ site/informacion/03_educacion.html

Banco Mercantil. (2011). Informe anual 2011. Disponible en http:// www.bancomercantil.com/mercprod/site/inversionistas/ historicos/banco_mercantil/informes_anuales/esp/MBU_ Informe_Anual_2011.pdf

Banesco. (2011). Informe segundo semestre 2011. Disponible en http:// www.banesco.com/pdf/informe_2do-sem_2011.pdf

Brugmann, J. y Phahalad, C. (2007). Cocrear un nuevo pacto social para las empresas. Harvard Business Review (América Latina), agosto, 80-90.

Coca-Cola FEMSA. (2009). Visión Global. Disponible en http:// www.femsa.com/es/social/global-vision/venezuela.htm

Comisión Europea. (2001). Libro Verde. Fomentar un marco europeo para la responsabilidad social de las empresas. Bruselas: Comisión de las Comunidades Europeas.

Comisión Europea. (1995). Libro Verde de la Innovación. Bruselas: European Commission Publications.

Comisión Europea. (2011a). Empowering people, driving chance. Social Innovation in the European Union. Luxemburgo: Publications Office of the European Union.

Comisión Europea. (2011b). Estado de la Unión por la Innovación 2011. Informe de la comisión al Parlamento Europeo, al Consejo, al Comité Económico y social europeo de las regiones. Luxemburgo: Publications Office of the European Union. Disponible en http://eur-lex.europa.eu/LexUriServ/ LexUriServ.do?uri=COM:2011:0849:FIN: es:PDF 
Comisión Europea. (2011c). Estrategia renovada de la UE para 20112014 sobre responsabilidad social de las empresas. Comunicación de la Comisión al Parlamento Europeo, al Consejo, al Comité Económico y social europeo de las regiones. Bruselas: Oficina de Publicaciones de la Unión Europea.

Culshaw, F. (2010). Negocios inclusivos en Venezuela. Debates IESA, 15(3), 32-35.

Fundación Andes Tropicales. (n.f.). Disponible en http://www. andestropicales.org/Proyectos.html

Fundación de la Innovación Bankinter. (2009). Innovación social. Reinventando el desarrollo sostenible. Disponible en http://www. fundacionbankinter.org/es/publications/social-innovationreinventing-sustainable-development

Fundación Polar. (n.f.). Disponible en http://www.fundacion empresaspolar.org/home/desarrollo-comunitario-3

Fundación Santa Teresa. (n.f.). Disponible en http://www.fundacion santateresa.org/fundhacemos.php?lang=esp

Fundación Telefónica. (n.f.). Disponible en http://www.fundacion. telefonica.com.ve/

Guedez, V. (2006). Ética y práctica de la responsabilidad social empresarial. El aporte de la empresa al capital social. Caracas, Venezuela: Editorial Planeta.

Guedez, V. (2008). Ser confiable. Responsabilidad social y reputación empresarial. Caracas, Venezuela: Editorial Planeta.

Harris, M. y Albury, D. (2009). The innovation imperative: Why radical innovation is needed to reinvent public services for the recession and beyond. London: NESTA.

Langlois, A. (2010). RSE y políticas públicas. Entre la cooperación y la compensación. Trabajo presentado en IX Cumbre Iberoamericana de Comunicaciones: Cambios en el escenario sociocultural del siglo XXI, Buenos Aires, Argentina.

Llano, A. (2004). Responsabilidad social empresarial e innovación. Revista valores en la sociedad industrial, 22(59), 66-75.

NESTA. (2008). Social innovation: New approaches to transforming public services. Policy Briefing, SI 18, January, 1-4. Disponible en: http:// www.nesta.org.uk/library/documents/social_innovation_pb.pdf 
OECD. (2010). Social entrepreneurship and social innovation. SMEs, Entrepreneurship and Innovation. Disponible en http:/ / ec.europa. eu/internal_market/social_business/docs/conference/oecd_ en.pdf

Organización de las Naciones Unidas. (1998). Agenda 21. Conferencia de Naciones Unidas de Río de Janeiro sobre Medio Ambiente y Desarrollo. Disponible en http://www.un.org/esa/sustdev/ documents/ agenda21/spanish/agenda21sptoc.htm

Organización para la Cooperación y el Desarrollo Económico (OECD) y Comunidad Europea. (2006). Manual de Oslo. Guía para la recogida e interpretación de datos sobre innovación. Tercera edición. Disponible en http://www.tragsa.es/filedcts/Manual_ de_Oslo.pdf

Petróleos de Venezuela S.A. (2009). Informe de gestión anual. Disponible en:www.pdvsa.com/interface.sp/database/fichero/ free/5889/1049.PDF

Phills Jr., J., Deiglmeier, K., y Miller, D. (2008). Rediscovering social innovation. Stanford Social Innovation Review, 6(4), 34-43.

Prahalad, C.J. (2006). The fortune at the bottom of the pyramid: Eradicating poverty through profits. NJ: Prentice Hall.

Red del Pacto Mundial. (n.f.). Pacto Mundial. Disponible en http:// www.pactomundial.org

Red Iberoamericana de Indicadores de Ciencia y Tecnología (RICYT) y Organización de Estados Americanos (OEA). (2001). Normalización de Indicadores de Innovación Tecnológica en América Latina y el Caribe. Manual de Bogotá. Disponible en http://www. ricyt.org/interior/difusion/pubs/bogota/bogota.pdf

Westall, A. (2007). How can innovation in social enterprise be understood, encouraged and enabled? A social enterprise think piece for the Office of the Third Sector. Cabinet Office. Office of the Third Sector. Disponible en www.cabinetoffice.gov.uk/ media/cabinetoffice/third_sector/ assets/innovation_social_ enterprise.pdf 
